In considering this question it should be remembered that the eye is capable of rude departures from recognised histological rules-we mention two of these-the dilator muscle arising from ectodermal tissue, and the growth throughout life of the lens, an ectodermal tissue, without ever casting off any of its cells.

I am indebted to Mr. H. B. Stallard for his kindness in allowing me to make free use of the pathological laboratory at Moorfields.

\title{
THE DIAGNOSIS OF IRIDO-CILIARY TUBERCULOSIS
}

\author{
BY \\ HENRI LAGRANGE* \\ CHIEF OPHTHALMOLOGIST, PARIS HOSPITAL SERVICES
}

BEFORE entering upon discussion of the report on the direct and differential diagnosis of tuberculosis of the iris and ciliary body, I desire to present this problem in a few words as illuminated by the papers which I have had the honour of classifying and abstracting for your information.

\section{I.-Ocular Tuberculosis is a Secondary Lesion supposedly due to Superinfection}

Publication of the first case of iridian tuberculosis by Gradenigo and Richetti, in 1867, was immediately followed by a discussion on the pathogenesis which has only recently been really closed. It was necessary to determine. whether irido-ciliary tuberculosis was a primary or a secondary effect.

Many collected cases seemed to indicate that this affection was primary. Denig, for example, collected, in 1885, 38 cases of localized ocular tuberculosis not having any evident previous history of tuberculosis. With these cases he compared systematic examination of 60 cases of pulmonary tuberculosis, 90 cases of bone tuberculosis, 20 cases of glandular tuberculosis and 20 cases of various types of tuberculosis. In all of these comparative cases, only five showed ocular metastasis.

In contrast with this finding, however, was the opinion stated by de Wecker, in France, Leber, in Germany, and Fuchs, in Austria,

* Introduction to discussion of my report on the direct and differential diagnosis of tuberculosis of the iris and ciliary body. International Congress on Ophthalmology, Madrid, April 16, 1933 . Cf. " Tuberculosis of the Iris and Ciliary Body," published by G. Doin \& Company, Paris, 1933. 
and based upon clinical evidence, that deep ocular tuberculosis originated from some other point in the organism and that it was consequently secondary. In 1905, differences in opinion were so marked that Schmidt-Rimpler wrote: "I confess that I do not understand why Fuchs and Leber disagree with Michel in believing that tuberculosis of the iris is always secondary and dependent upon a primary site of tuberculosis. Why may not the bacilli of tuberculosis reach the iris by way of the blood as well as they may reach bones and lymphatics, producing there the first visible signs of the affection ?"

This teaching became progressively affirmed by experimental study and agrees with the present views universally held in phthisiology, namely, that tuberculosis of the uveal tract arises from a deeper site not at first recognized.

Tuberculous infection is thus conveyed to the uveal tract through the blood, as believed by Leber. The first experimental demonstration of the fact was presented by Felix Lagrange, in 1898. Injecting an emulsion of tubercle bacilli into the carotid arteries of ten rabbits, he produced tuberculous infection of the uveal tract in two cases. These studies of his have established the basis of our present pathogenic knowledge on the question. They were repeated, verified and completed nine years later by Stock and Daels (1907) and mark one of the historic stages in scientific progress, since they show the manner in which tuberculous infection reaches the uveal tract, by a bacillaemic or a septicaemic process. I believe that it is now possible to determine from Aschoff's work on the reticuloendothelial system why the uveal tract constitutes a favourable site for the formation of tubercles.

While the work by Felix Lagrange, Daels and Stock concerning the mechanism of localization of tuberculosis in the uveal tract proves its haemosporic origin, it was evident that these writers obtained only miliary lesions not possessing the characters of uveal tuberculosis commonly present clinically. For this reason I attempted to obtain, in 1923, experimental specimens more like those occurring in human pathology. I succeeded in this endeavour by inoculating the uveal tract from animals previously rendered tuberculous. My results formed a contrast with the local reactions following primary inoculation and with the lesions obtained with superinfection, that is, by inoculating with tuberculous products the uveal tract of animals previously rendered tuberculous. These studies showed that it is necessary to use animals previously rendered allergic in order to reproduce tuberculous lesions of the uveal tract which resemble those encountered clinically.

Onward from this point, ophthalmological cases accumulating for nearly fifty years may be interpreted according to the principles of general pathology which, especially influenced by the work of 
Ranke, have shown that the wide variations occurring in tuberculous lesions are due to variations in allergy. Even if these various data be considered only from the diagnostic viewpoint, a fundamental idea is derived from them, namely, that irido-ciliary tuberculosis, even when occurring as an isolated sign in an organism appearing to harbour no other localization of the tuberculous infection, is derived from a primary site, which demands initial study and examination.

\section{II.-Diagnosis requires initial proof of the presence of Primary Infection}

If it be desired to state just when it was perceived that ocular tuberculosis develops in individuals not presenting other visceral signs of progressive tuberculosis, it suffices to reconsider the history of the pathogenic controversy alluded to by Schmidt-Rimpler and entered into by such men as Fuchs, Leber, Parinaud or von Michel. Clinicians of such eminence believed that tuberculosis may be primary in the irido-ciliary region because ocular tuberculosis rarely co-exists with distinct signs of previous tuberculous infection.

(a) Pulmonary signs. - In this report I have reviewed the history of the cases accumulated on this point, and, like Rist, have been obliged to conclude that "pulmonary forms of tuberculosis, on the one hand, and extra-pulmonary manifestations, on the other, form two distinct groups, since they present more than one contrasting character and exhibit a singular sort of balance."

Though extra-pulmonary forms are characterized by being clinically primary, though their activity is in nowise parallel to that of pulmonary lesions, and though it is vain to seek concomitant pulmonary lesions for the diagnosis of the extra-pulmonary forms, are there no guiding or orienting points in the general examination of a case which should be learned and sought for?

J. Meller seeks pulmonary signs in every case of ocular tuberculosis, and stresses the frequency with which recurrent pleuritis is encountered. Rist and most contemporary writers consider that ocular tuberculosis is always secondary to a pre-existing pulmonary site of infection, but if the latter produces no noticeable signs, this "chancre of primary inoculation" may always be shown by careful $\mathrm{X}$-ray examination. No better description is possible than that given by Rist and Ribadeau-Dumas; who say, "these calcified vestiges of cured primary tuberculous inoculation occur in the radiograms of healthy children or adults. They usually occur at the base of a lobe, often in the prolongation of a bronchial twig, sometimes very near the pleura in the axillary region and rarely at the pulmonary apices. Whenever such an intra-parenchymatous 
calcification is discovered, a homologous calcification may always be found in the pulmonary hilum, and consequently in a gland."

(b) Cardio-vascular signs.-It goes without saying that other regions of the organism (digestive tract, bones, joints and lymphatic system) should also be thoroughly examined, but I desire to stress the importance which I personally attach to study of the cardiovascular system. Tuberculous infection may attack this system as it may invade any other structure, especially producing tuberculous cardiopathies. The latter, however, are not important from our present viewpoint, for we are more particularly interested in the socalled "tuberculous heart," which expresses how tuberculous infection, localized in some particular structure, especially affects the cardio-vascular apparatus.

The tuberculous heart is characterized by three signs, namely, by tachycardia, microcardia and hypotension. These signs may differ in degree and are not determined by pulmonary localization of the infection. They may favour or promote it, but they are not secondary to changes in the haematosic field. They are the consequence of tuberculous impregnation. I wish especially to stress the significance of hypotension, for Potain and Teissier considered it very useful for differentiating true forms of chlorosis not affecting blood pressure from the chlorotic conditions symptomatic of tuberculosis.

The presence of tuberculosis should naturally not be judged solely from the presence of hypotension, but the facility with which the latter may be measured, under ordinary clinical conditions, constitutes it a clinical test which should be applied to all doubtful cases, all cases of irido-cyclitis, for example, not presenting convincing reasons for being considered syphilitic. I am fully aware that hypotension occurs frequently, especially in young people, and that it is nearly constantly present in certain nontuberculous affections, such as typhoid or para-typhoid, or infection with b. coli. I do not claim at all that it is a pathognomonic sign, but that it is a necessary sign. In the presence of irido-cyclitis of doubtful nature, the origin is probably not tuberculous if hypotension is absent. Here probability must be largely allowed for. Just as essential glaucoma never occurs during phthisis, blood pressure is never high in tuberculosis save in the presence of nephritic conditions, of special type. These diagnostic elements are independent of rules. They are more particularly derived from custom, and the latter formulates the law in pathology, as in other sciences.

(c) Humoral signs.--The fact that ocular tuberculosis proves to be secondary tuberculosis renders it difficult from the viewpoint of aetiological diagnosis, to utilize the data accumulated on this point as a result of biological studies. Attempts to utilize them in 
ophthalmology should be respected. They are numerous, and, just as I was writing that the Vernes reaction proves useful in ophthalmology, my colleague Lacroix, of Rouen, was publishing an article presenting a large number of facts. When the humoral test proves the presence of tuberculosis, however, the aetiological diagnosis of the ocular lesion is not established. Is not the occurrence of a cuti-reaction positive to tuberculin entirely a normal sign?

\section{III.-The Method of Choice}

However, should all of this work be discarded? Are so many attempts wholly vain? Extreme pessimism should be avoided. Three special methods appear already to be highly promising, as follows :-

1. Tuberculin diagnosis, applied according to the given case either in the form of test treatment or in the form of the homeotopic technique of Marc Weiss.

2. Micro-leucocytic culture, as employed by Löwenstein.

3. Search for the ultra-virus of tuberculosis by the intraglandular inoculation of guinea pigs.

Tuberculin diagnosis, in cases presenting reactions positive to tuberculin, may supply absolutely decisive findings referable to focal reactions (at the affected site) in the course of test treatment with tuberculin. In cases in which the reaction is negative, the homeotopic method of Marc Weiss may give qualitative indications (according to the expression of Werdenberg) concerning the allergic condition present. When the reaction becomes negative, the reaction should be tested at the original site. If the patient is not sensitized to tuberculin, he is not tuberculous. If he becomes sensitized after a long time, his reactivity is low, and in other words, his immunity is diminished.

No conclusions are warranted from the work by Löwenstein, of Vienna. This work presents intensely interesting cases referring to the frequency with which the tubercle bacillus may be found in the blood during various diseases, but these studies are as yet incomplete and it is not yet practicable to define the field of so-called "emergent" tubercle bacilli or the pathogenic significance of such bacilli.

Search for the tuberculous ultra-virus seems to merit attention on account of recent work on tuberculous rheumatism suggested by the labours of Antonin Poncet and also on account of the facility with which, by simple puncture of the anterior chamber of the eye, it is possible to make aetiological verification by inoculating the glands of guinea pigs. 


\section{IV.-The Morphological Diagnosis of Irido-ciliary Tuberculosis}

At the site of the irido-ciliary diaphragm, the characters peculiar to the development of tuberculosis are naturally associated with signs supplied by the anatomy and physiology of the special structures which are affected. These include a reservoir containing differentiated fluids, in mysterious equilibrium, and a muscular diaphragm of delicate texture, designed for adaptation and accommodation. These peculiarities determine special signs which are independent of the specificity of the infecting bacterium. Here tuberculosis develops in a mesenchymatous system. Whether productive or exudative, it is bathed by the interstitial liquid and develops amid muscular fibres and glandular structures. In short, it finds, on a narrow surface, the structural elements favourable to the production of lesions which may also occur in the pleura, serous membranes of the joints, or the pulmonary or glandular tissue, but never occur in such a variety in such a tiny space in any other part of the organism.

The wide variety of the clinical forms of irido-ciliary tuberculosis is quite familiar. The reporter has not been requested to describe these forms and he naturally limits his work to the production of an outline which may be easily filled from the copious data readily available. In completing the task with which he has been honoured, he has naturally sought to present clearly established points. There can be no disagreement with the thought that strict and close analysis is essential in any work of value and that all unknown quantities should be excluded from discussion.

The masters in medicine who lived during the epoch-making work of Pasteur and the discoveries of Villemin and Robert Koch have accomplished their tasks. They completed clinical and bacteriological investigations. They have perfected descriptions and created methods of examination tending to establish the specific characters possessed by various diseases. Whatever the anatomicoclinical form assumed by irido-ciliary tuberculosis, the lesions which we have studied result from a number of different factors. Some of these refer to the bacillus and others to conditions of the local tissues. Clinical facts, experimental findings and studies in allergy show that even an identical specitic substance (bacillary or tuberculous products) capable of releasing a reaction produces in different tuberculous individuals, or in the same individual at different periods of his disease, reactions which are different. This fact already suffices to explain in large measure the variety of pathological reactions which may occur.

But Bordet pointed out, at the International Tuberculosis Conference held at the Hague, in 1932, that a positive cuti-reaction 
may be obtained with a tuberculous organism by means of a filtrate of $b$. coli, which is entirely identical with the reaction produced by tuberculin in the same organism. Bezançon notes in his address given at the Twenty-second French Congress on Medicine that the substance extracted from a culture-free glycerinated bouillon gives a cutaneous reaction with tuberculous individuals which is analogous with that produced by tuberculin. A tuberculous individual may thus react identically either to specific substances, such as tuberculin, or to non-specific substances, such as a b. coli filtrate or even an extract of a culture-free glycerinated bouillon. Such data show most strikingly how complex is the idea of specificity.

\section{V.-Conclusion}

A study of the diagnostic methods which I have examined thus indicates certain rules of procedure, but uncertainties still remain and must persist until there is fuller information concerning the reciprocal influences of diatheses, hereditary dystrophies, congenital infectious diseases and acquired infections. Venneman frankly admitted inability to determine the part played by gout, tuberculosis and hereditary syphilis in the persistent form of iritis observed by Hutchinson in children born of gouty parents. Such questions are not solved as yet. In the general problem concerning morbid specificity, the question of associated affections, such as scrofula and syphilis, requires explanation.

When these problems become clear, and when clinical work shall be completed by knowledge of the pathogenic conditions which are still obscure in irido-ciliary tuberculosis, we shall render due respect to the pleas which have been faithfully made in directing attention to the frequency of tuberculous irido-cyclitis. In this department of tuberculosis, in which the truth develops only incompletely and in a fragmentary way, and in which observed facts supply no complete solutions, it is as yet too soon to generalize. Much, however, has been accomplished in obtaining so many useful data and in finding the direction which studies and research should take.

\section{ANNOTATION}

\section{The Role of Filtration in the Maintenance of the Intra-ocular Pressure}

In the forward march of any scientific work with its penetration and conquest of new fields, its changing direction of advance and the varying phases of its encounters, it is at times desirable for the participants to stand apart and review in panorama the ground over 